\title{
A National Audit of Current Cardiac Device Policies from Radiotherapy Centres across the UK
}

\author{
J.F. Lester* ${ }^{*}$, L.M. Evans $*$, Z. Yousef $\dagger$, A. Penney $\dagger$, P.N. Brown $\ddagger$, R. Perks $\ddagger$ \\ * Velindre Cancer Centre, Cardiff, UK \\ ${ }^{\dagger}$ Cardiology Department, University Hospital of Wales, Cardiff, UK \\ ${ }^{\ddagger}$ Cardiff University, Cardiff, UK
}

Received 10 April 2013; received in revised form 2 September 2013; accepted 3 September 2013

\begin{abstract}
Aims: The number of patients with cardiac implantable electronic devices (permanent pacemakers and implantable cardioverter defibrillators) undergoing radiotherapy treatment is increasing. The aims of this audit were to establish current UK practice regarding the management of patients with implanted cardiac devices undergoing radiotherapy and to compare this practice with current 'gold standard' evidence-based guidelines.

Materials and methods: All UK radiotherapy departments were contacted and asked to provide their current cardiac implantable electronic device policy or to indicate if there was no current policy. A proforma was created to analyse these polices and to compare with current best practice.

Results: In total, 47/67 (70\%) radiotherapy departments responded and 45 departmental policies were submitted; $31 / 45$ (69\%) policies defined the radiotherapy tolerance dose to permanent pacemakers and $14 / 45$ (31\%) defined the monitoring procedure for patients in line with current best practice. Only $5 / 45$ (11\%) policies defined the radiotherapy tolerance dose to implantable cardioverter defibrillators and $12 / 45$ (27\%) defined the monitoring procedure in line with current best practice.

Conclusion: Most UK cardiac device policies do not reflect current best evidence. Policies are based on research carried out in 1994 by the American Association of Physicists in Medicine. This evidence does not account for advances in cardiac implantable electronic device technology. Further research is urgently needed to establish the effect of radiotherapy on these devices.

(c) 2013 The Royal College of Radiologists. Published by Elsevier Ltd. All rights reserved.
\end{abstract}

Key words: Cardiac device; defibrillator; national survey; pacemaker; policy; radiotherapy

\section{Introduction}

The life expectancy of the English and Welsh population has increased by more than $65 \%$ in the past century [1]. This has resulted in a higher prevalence of cardiovascular morbidity, leading to an increase in the number of patients with cardiac implantable electronic devices (CIEDs) [2,3]. In addition, the age-standardised incidence of cancer has increased by more than $25 \%$ in the past 30 years [1]. It has been estimated that $50-60 \%$ of all patients with cancer will require radiotherapy during the course of their illness [4]. Therefore, with an ageing population and an increase in the incidence of both cardiovascular morbidity and cancer, the number of patients with CIEDs presenting for radiotherapy treatment will probably increase $[2,5,6]$.

Author for correspondence: J. Lester, Velindre Cancer Centre, Velindre Road, Whitchurch, Cardiff CF14 2TL, UK. Tel: +44-292-0196159.

E-mail address: Jason.Lester2@wales.nhs.uk (J.F. Lester).
There are two categories of CIED fitted into patients; permanent pacemakers and implantable cardioverter defibrillators (ICDs). Permanent pacemakers are referred to as 'pacemakers' in this paper. Pacemakers are permanent devices and vary in sophistication. ICDs are more sophisticated devices and have the ability to automatically defibrillate the heart by monitoring the patient's heart rate and delivering the appropriate electrical therapy.

Although most medical treatments pose little danger to the functioning of CIEDs, radiotherapy has the potential to cause device malfunction. Cardiac devices may be affected in two ways; electromagnetic interference (EMI) and direct damage to the circuitry via ionising radiation, both of which may cause temporary and permanent device malfunction [3]. Changes within the device parameters as result of EMI are seen even when the CIED is placed outside the radiotherapy treatment field [7].

Over the past three decades, the design and technology of CIEDs has evolved and the use of complementary metal 
oxide semiconductor circuits has expanded [8]. These are more sensitive to ionising radiation than bipolar semiconductor devices used previously, possibly resulting in increased damage and catastrophic failure of the cardiac conduction system in the device $[9,10]$.

It is not possible to predict the exact behaviour of a CIED when it is in or close to the radiotherapy treatment field [11]. In addition, published results are not consistent in their findings or recommendations. Radiotherapy has been shown to cause malfunction of CIEDs, ranging from inappropriate triggering, device reprogramming or device failure [12-14]. Other investigators have reported a minimal effect of radiotherapy on CIEDs [15].

There is concern that the photon energy of the treatment beam may also be important. Gelblum and Amols [16] discussed the possible effects of neutron contamination from high-energy photon beams. They recommended the use of low-energy beams ( $<10 \mathrm{MV}$ ), but there is little evidence to support this recommendation.

The American Association of Physicists in Medicine (AAPM) [17] published a report in 1994 on the safe use of radiotherapy in patients with permanent pacemakers. The AAPM report is the basis of most of the current CIED departmental radiotherapy policies in the UK (authors' observation). Frizzell [18] published a more contemporary review and a distinction was made between pacemakers and ICDs. Both the AAPM and the Frizzell reports are widely referenced in the literature and in our opinion have the most robust evidence base to support them. Despite this, the AAPM report is now nearly two decades old and does not reflect advances in CIED or radiotherapy technology. However, in the absence of more contemporary evidencebased guidelines on treating CIED patients with radiotherapy, it is reasonable to compare current UK policies with the AAPM recommendations, using the Frizzell update to define current best practice for ICD management.

Below is a summary of the AAPM and Frizzell recommendations:

AAPM Recommendations (pacemakers):

1. Pacemakers should not be placed in the direct (unshielded) therapy beam.

2. The absorbed dose to be received by the pacemaker should be estimated before treatment and limited to $2 \mathrm{~Gy}$.

3. If the total estimated dose to the pacemaker might exceed $2 \mathrm{~Gy}$, pacemaker function should be checked before radiotherapy and possibly at the start of each following treatment week by a cardiologist.

4. Patients should be closely observed during the first radiotherapy treatment on a linear accelerator.

Frizzell Recommendations (ICDs):

1. The absorbed dose to be received by the ICD should limited to $0.5 \mathrm{~Gy}$.

2. A magnet should be placed over an ICD when a patient is exposed to radiation.

3. Notify all patients about the possibility of ICD malfunction, failure or both.
Monitoring recommendations:

1. Patients should be monitored with a continuous electrocardiogram (ECG) strip during the first radiotherapy treatment. This strip should then be reviewed for any evidence of pacing disruption when radiotherapy is being administered.

2. ICD patients should undergo daily monitoring and staff should document any changes in the patient's physical status and any changes in the ECG trace.

3. Monitoring should be carried out by fully trained and competent health professionals. If therapeutic radiographers are monitoring patients, they should receive specific training on the management and monitoring of these patients.

4. If at any point malfunction is suspected or detected, the clinical oncologist and cardiologist should be immediately informed.

Consent recommendations:

1. The patient is aware of potential adverse effects of radiotherapy on CIEDs.

2. The patient is aware the ICD will be deactivated during radiotherapy.

Currently, there are no UK or national guidelines on the use of radiotherapy in patients with CIEDs and most radiotherapy departments have no formal risk management strategy or policy in place [11]. The aims of this audit were to establish current UK practice regarding the management of patients with CIEDs undergoing radiotherapy and to compare this practice with the current 'gold standard' AAPM and Frizzell recommendations.

\section{Materials and Methods}

UK radiotherapy centres were identified using the Society and College of Radiographers' database. Between May 2012 and March 2013, all radiotherapy department managers were e-mailed asking them to participate in a national audit. Centres were asked to either provide their current CIED policy or to indicate if there was no policy.

A proforma was created to analyse CIED policies comprising two sections: first, the roles and responsibilities of healthcare professionals; second, treatment and management guidelines. All data collected were anonymised. A database (Microsoft Excel) was created for the entry and analysis of audit data and departmental guidelines were compared. The results are presented as simple frequencies and percentages.

\section{Results}

In total, 67 radiotherapy centres were identified in the UK and contacted. Overall, 47/67 (70\%) departments responded to the request to provide their policy for inclusion in the audit. Forty-five departments provided their policy and two 
departments are currently re-writing their CIED policy and were excluded from the results. Twenty departments did not respond to the request. The analysis was carried out on the 45 polices submitted that are currently in use.

Table 1 summarises the roles and responsibilities of healthcare professionals. Overall, 39/45 (87\%) policies require the clinical oncologist to state whether a CIED is present on the radiotherapy referral form; $41 / 45$ (91\%) policies require the clinical oncologist to contact the patient's cardiology department before starting radiotherapy; $34 / 45$ (76\%) policies require a cardiology assessment; 36/45 $(80 \%)$ policies require the clinical oncologist to provide relevant information to medical physics to allow the calculation of the estimated cumulative dose to the CIED before starting radiotherapy; $12 / 45$ (27\%) policies require patients fitted with CIEDs to be informed of the risks to themselves and their device before starting radiotherapy; 5 / $45(11 \%)$ policies state that patients fitted with ICDs should be informed about the possibility of ICD malfunction or failure during radiotherapy and must give consent to deactivate the ICD during radiotherapy.

Of note is that in only $29 / 45$ (64\%) policies is it mandatory for the planning radiographer to contact the treating consultant if the CIED is within a radiotherapy treatment field or the estimated dose is too high. Less than a third of policies include appropriate monitoring procedures for treatment radiographers in patients with pacemakers or ICDs. There is no requirement for medical physics to calculate the estimated dose to CIEDs and leads in 9/45 (20\%) policies.
Table 2 summarises adherence to current guidelines: $31 /$ $45(69 \%)$ policies define the radiotherapy tolerance dose to the pacemaker recommended in the AAPM guidelines. Of these, 21/31 (68\%) policies require the cardiology department to be contacted to discuss safe management of the patient. In total, $14 / 45$ (31\%) policies do not define a tolerance dose limit to the pacemaker and none of these radiotherapy departments contact the patient's cardiology department. Only 5/45 (11\%) policies define the radiotherapy tolerance dose limit to the ICD as $0.5 \mathrm{~Gy}$ and all five of these radiotherapy departments contact the cardiology department to discuss the management of the patient; 23/ $45(51 \%)$ policies define a dose limit of $>0.5 \mathrm{~Gy}$ to the ICD and $17 / 45$ (38\%) policies do not define a dose limit. 39/40 (98\%) policies that use an incorrect tolerance dose limit or do not define a dose limit do not mandate contacting the patient's cardiology department for advice. 30/45 (67\%) policies require a follow-up appointment to be made with the patient's cardiology department after the completion of radiotherapy.

Table 3 summarises the monitoring requirements for patients with CIEDs receiving radiotherapy. Overall, 31/45 (69\%) policies do not define monitoring procedures for patients with pacemakers in line with the AAPM guidelines and none mandate the use of appropriately trained staff to monitor patients. Similarly, 33/45 (73\%) policies do not define monitoring procedures for patients with ICDs in line with the Frizzell report and none mandate the use of appropriately trained staff to monitor patients.

Table 1

Roles and responsibilities

Results ( $n=45$ unless stated otherwise)

Number of radiotherapy \% department policies

\section{Consultant oncologist}

Identify patient's CIED status and highlight on radiotherapy referral form

Contact patient's cardiology department before starting radiotherapy treatment

Request cardiology assessment

Provide medical physics with information to calculate cumulative radiotherapy dose to CIED

Dose to the implantable internal pacemaker does not exceed $2 \mathrm{~Gy}$

Dose to the ICD does not exceed 2 Gy

Consent - patient aware of potential adverse effects of radiotherapy on cardiac device

Consent - switch off ICD during radiotherapy

Planning radiographers

Annotated patient's CIED status

CIED included in scan if in/close to the radiotherapy treatment field

Medical physics informed of patient's CIED status

Contact consultant if CIED is within radiotherapy treatment field or estimated dose too high

Treatment radiographers

Appropriate monitoring procedure for patients with pacemakers 
Table 2

Adherence to American Association of Physicists in Medicine (AAPM) and Frizzell guidelines

\begin{tabular}{|c|c|c|}
\hline \multirow[t]{2}{*}{ Guidelines } & \multicolumn{2}{|c|}{$\begin{array}{l}\text { Results ( } n=45 \text { unless stated } \\
\text { otherwise) }\end{array}$} \\
\hline & $\begin{array}{l}\text { Number of radiotherapy } \\
\text { department policies }\end{array}$ & $\%$ \\
\hline \multicolumn{3}{|l|}{ Pacemakers - AAPM guidelines } \\
\hline 2 Gy radiotherapy tolerance dose to device stated & 31 & 69 \\
\hline Requirement to contact cardiology if radiotherapy dose to pacemaker exceeds $2 \mathrm{~Gy}$ & $21($ of 31$)$ & 68 \\
\hline Cardiology follow-up made after radiotherapy completed & 30 & 67 \\
\hline \multicolumn{3}{|l|}{ ICDs - Frizzell Report } \\
\hline 0.5 Gy radiotherapy tolerance dose to device stated & 5 & 11 \\
\hline Requirement to contact cardiology if radiotherapy dose to ICD exceeds $0.5 \mathrm{~Gy}$ & 5 (of 5$)$ & 100 \\
\hline 1 Gy radiotherapy tolerance dose to device stated (exceeding $0.5 \mathrm{~Gy}$ tolerance dose) & 9 & 20 \\
\hline Do not contact cardiology if radiotherapy dose to ICD exceeds $1 \mathrm{~Gy}$ & 9 (of 9$)$ & 100 \\
\hline Cardiology follow-up made after radiotherapy completed & 30 & 67 \\
\hline
\end{tabular}

ICD, implantable cardioverter defibrillator.

\section{Discussion}

The number of patients with CIEDs undergoing radiotherapy treatment is increasing $[2,5,6]$. There is limited published research on the effect of radiotherapy on CIEDs, but there is evidence to show radiotherapy at low doses can cause malfunction or failure with potentially lifethreatening consequences [10]. Given this risk, all radiotherapy centres should have policies in place to support the safe radiotherapy treatment of patients with CIEDs.

This audit used the AAPM guidelines and Frizzell report as the benchmark to compare UK radiotherapy departments' current CIED policies, as in the opinion of the authors, these guidelines had the most robust evidence base to support them $[17,18]$.
The first question we wanted to answer was how many UK radiotherapy centres have a CIED policy in routine use. All radiotherapy department managers were asked to provide their current policy for analysis. The request yielded a response rate of 70\%; 45 respondents provided their policy and two centres stated that they are currently re-writing their policy. A third follow-up e-mail has been sent from the Society of Radiographers on behalf of the researchers asking radiotherapy departments to forward their policy or to inform the researchers if they do not have one. This will allow a more detailed national picture to be established in the future, but a response rate of 70\% was felt high enough to proceed with this review. At this point, it is not known whether the remaining 20 radiotherapy departments who have not responded have a policy, but it is possible that up

Table 3

Monitoring

Clinical practice - monitoring of patients with cardiac devices

Pacemakers - AAPM guidelines

Appropriate monitoring procedure

Appropriate staff used to monitor patients

Close observation of patient using cardiac monitor on first fraction of radiotherapy

Subsequent monitoring requirements assessed and annotated

ICDs - Frizzell report

Appropriate monitoring procedure

Appropriate staff used to monitor patients

12 lead continuous strip ECG before first fraction of radiotherapy

Deactivate ICD with magnet during radiotherapy

Continuous strip ECG monitoring for all subsequent treatments

Document any change in patient's status
Results ( $n=45$ unless stated otherwise)

Number of radiotherapy \%

department policies

$\begin{array}{ll}14 & 31 \\ 14 & 31 \\ 14 & 31 \\ 14 & 31 \\ 12 & \\ 12 & 27 \\ 12 & 27 \\ 12 & 27 \\ 12 & 27 \\ 12 & 27 \\ \end{array}$

AAPM, American Association of Physicists in Medicine; ECG, electrocardiogram; ICD, implantable cardioverter defibrillator. 
to $30 \%$ of UK radiotherapy centres have no policy for managing patients with CIEDs. Given the potential risk from radiotherapy to patients with CIEDs, it is concerning that a significant proportion may not have a policy to guide healthcare professionals. The lack of an over-arching national policy on this therapy area is not specific to the UK. An American report suggests that $12 \%$ of US oncology departments have neither a formal risk management strategy nor a cardiac device policy and that only $15 \%$ have a written policy [11].

The audit results highlight significant differences between policies in the roles and responsibilities of healthcare professionals involved in the patient pathway and the management of patients with a CIED receiving radiotherapy. From the results of the audit, in $87 \%$ of radiotherapy departments the treating clinical oncologist determines CIED status and highlights it on the radiotherapy referral form. This means that in $13 \%$ of the policies included in this review, it is left to radiographers to identify whether a CIED is present. Anecdotal evidence from this audit shows that in some cases, a CIED is not discovered until a patient attends for radiotherapy. This results in treatment being delayed or treatment proceeding without safety measures in place. It is not known how many patients with CIEDs undergo radiotherapy without the knowledge of the therapeutic radiographers, but this potentially dangerous scenario is less likely if the treating oncologist determines early on in the treatment pathway that a device is present and informs the planning and treatment teams. Worryingly, in only $29 / 45$ (64\%) policies is it mandatory for the treating consultant to be contacted if the CIED is within a radiotherapy treatment field or the estimated dose is too high. In most cases, this communication would probably happen even in the absence of policy. However, given the potential harm to the patient, this should be explicit. There is clearly a need for policies in use to include monitoring procedures for treatment radiographers in patients with pacemakers or ICDs. These procedures are currently included in less than one-third of policies and it is vital that patients having treatment are monitored to minimise the chance of harm. There is no requirement for medical physics to calculate the estimated dose to CIEDs and leads in 9/45 (20\%) policies. Without this estimation being made before radiotherapy starts, patients may be exposed to doses of radiation that exceed the limits recommended by AAPM and Frizzell.

Patients consenting for any type of treatment need to be informed of potentially serious side-effects related to that treatment. Nearly three-quarters of policies do not mandate discussion of potential damage to the CIED during and after radiotherapy in the treatment consent process. Given the lack of contemporary research in this area, it is not possible to quantify this risk of damage or harm at present, but consideration should be given to discussing potential complications in all patients with a CIED. ICDs are probably susceptible to radiotherapy damage at lower doses than pacemakers and ICD patients should be informed about the possibility of malfunction or failure during radiotherapy treatment as the consequences may be devastating. ICD patients also need to be told in advance of radiotherapy that their device will be deactivated using a magnet during treatment.

The AAPM report recommended that the cumulative radiotherapy dose to the pacemaker be limited to less than 2 Gy [17]. In the audit, only 31/45 (69\%) radiotherapy departments limit the cumulative dose to the pacemaker to $2 \mathrm{~Gy}$ and of these, only $21 / 31$ (68\%) require communication with the cardiology department if the dose exceeds $2 \mathrm{~Gy}$. It is concerning that nearly a third of policies define no tolerance dose to the pacemaker. There is evidence that even low cumulative doses of radiotherapy may damage CIEDs and patients are probably being put at risk of harm with the current policies in use.

The Frizzell report recommended a lower radiotherapy tolerance dose of $0.5 \mathrm{~Gy}$ for ICDs and that they should be deactivated before radiotherapy by placing a magnet over the device to prevent inappropriate therapy or shock delivery as a result of accidental sensing of EMI interference [18]. Worryingly, the audit shows that only 5/45 (11\%) radiotherapy departments limit the ICD dose to $0.5 \mathrm{~Gy}, 23$ / $45(51 \%)$ radiotherapy departments specify a higher ICD tolerance than recommended and $17 / 45$ (38\%) do not state a radiotherapy tolerance dose. That means that in most centres with a cardiac device policy, ICDs are potentially exposed to doses of radiotherapy that may affect function and cause serious harm to the patient.

In addition, it is of significant concern that only $6 / 45$ (13\%) CIED policies differentiate between pacemakers and ICDs and subsequently apply radiotherapy tolerance dose limits to both types of device. In these policies, ICDs are subject to the same radiotherapy tolerance dose limits and the same monitoring procedures as pacemakers. As a result, ICDs are almost certainly being subjected to radiotherapy doses beyond tolerance and ICD malfunction has potentially life-threatening consequences.

The AAPM and Frizzell reports recommend that all patients with CIEDs be monitored with a continuous ECG strip during their first radiotherapy treatment for any evidence of pacing disruption $[17,18]$. In addition, they should be monitored by an appropriately trained health professional. The audit shows that over two-thirds of policies do not mandate the monitoring procedures defined by AAPM and Frizzell and less than one-third require the use of an appropriately trained health professional to carry out the monitoring. Therefore, a significant number of patients with CIEDs are undergoing radiotherapy with no monitoring and in those that are monitored most of the staff involved may not have appropriate training to interpret ECG or clinical changes.

\section{Conclusion}

This audit of CIED policies is based on a 70\% response rate from radiotherapy centres in the UK. It cannot be definitive in its conclusions, but important themes have emerged nevertheless. It is clear that policies differ between radiotherapy centres. In addition, a significant proportion of 
policies do not adhere to current established tolerance doses for CIEDs. As a consequence, it is very likely that patients are being put at significant risk of harm. We are carrying out urgently needed research to further define the effect of radiotherapy on modern cardiac devices. This research will underpin the development of contemporary evidence-based guidelines on the use of radiotherapy in patients with these devices.

\section{References}

[1] Office for National Statistics. Mortality in England and Wales: average life span. Available from: http://www.ons.gov.uk/ons/ rel/mortality-ageing/mortality-in-england-and-wales averagelife-span/rpt-average-life-span.html [accessed March 2013].

[2] Kalache A, Keller I. The greying world: a challenge for the twenty-first century. Sci Prog 2002;83:33-54.

[3] Last A. Radiotherapy in patients with cardiac pacemakers. $\mathrm{Br} J$ Radiol 1998;71:4-10.

[4] National Radiotherapy Advisory Group. Radiotherapy: developing a world class service. National Radiotherapy Advisory Group; 2007.

[5] Brooks N, Norell M, Hall J, et al. Working group of the British Cardiac Society. National variations in the provision of cardiac services in the United Kingdom. Br J Cardiol 2005;12:192-198.

[6] Boon N, Norell M, Hall J, et al. National variations in the provision of cardiac services in the United Kingdom: second report for the British Cardiac Society Working Group. Heart 2006;92:873-878.

[7] Ferrara T, Baiotto M, Malinverni G, et al. Irradiation of pacemakers and cardio-defibrillators in patients submitted to radiotherapy: a clinical experience. Tumori 2010;96:76-83.

[8] Hurkmans CW, Knegjens JL, Oei BS, et al. Management of radiation oncology patients with a pacemaker or ICD: a new comprehensive practical guideline in The Netherlands. Radiother Oncol 2012;7:198.

[9] Little FA. Pacemakers in radiotherapy. Clin Oncol 1994;6:211-212.

[10] Mouton J, Haug R, Bridier A, Dodinot B, Eschwege F. Influence of high-energy photon beam irradiation on pacemaker operation. Phys Med Biol 2002;47:2879-2893.

[11] Solan AN, Solan MJ, Bednarz G, Goodkin MB. Treatment of patients with cardiac pacemakers and implantable cardioverter-defibrillators during radiotherapy. Int J Radiat Oncol Biol Phys 2004;59:897-904.

[12] Hurkmans CW, Scheepers E, Springorum BG, Uiterwaal H. Influence of radiotherapy on the latest generation of implantable cardioverter-defibrillators. Int J Radiat Oncol Biol Phys 2005;63:282-289.

[13] Hoecht S, Rosenthal P, Sancar D, et al. Implantable cardiac defibrillators may be damaged by radiation therapy. J Clin Oncol 2002;20:2212-2213.

[14] Niehaus M, Tebbenjohanns J. Electromagnetic interference in patients with implanted pacemakers or cardioverter-defibrillators. Heart 2001;86:246-248.

[15] Makkar A, Prisciandaro J, Agarwal S, et al. Effect of radiation therapy on permanent pacemaker and implantable cardioverter-defibrillator function. Heart Rhythm 2012;9:1964-1968.

[16] Gelblum DY, Amols H. Implanted cardiac defibrillator care in radiation oncology patient population. Int J Radiat Oncol Biol Phys 2009;73:1525-1531.

[17] Marbach JR, Sontag MR, Van Dyk J, Wolbarst AB. Management of radiation oncology patients with implanted cardiac pacemakers: report of AAPM Task Group No. 34. American Association of Physicists in Medicine. Med Phys 1994;21:85-90.

[18] Frizzell B. Radiation therapy in oncology patients who have a pacemaker or implantable cardioverter-defibrillator. Comm Oncol 2009;6:469-471. 